\title{
CHRONIC PROLIFERATIVE OSTEITIS OF THE SKULL
}

\author{
BY
}

\author{
J. E. M. DUTTON and G. L. ALEXANDER
}

From the Department of Neurological Surgery, Frenchay Hospital, Bristol

Three cases of this condition have come to our notice in recent years; in two of them the process was related to a chronic extradural abscess. Our purpose in this report is to describe our experience with these cases and to indicate the lessons which we believe may be learnt from them.

\section{Case Reports}

Case 1.-This patient was a man of 42 years. Twentyone years before, a left posterior frontal abscess had appeared spontaneously under the scalp. The abscess ruptured after three weeks, and thereafter a chronic sinus discharged intermittently. Local surgical exploration was undertaken on three occasions at long intervals, at the latest of which, bone an inch thick was chiselled away, releasing some extradural pus. The sinus continued to discharge, and the patient noticed that coughing and straining on occasion caused as much as an ounce of pus to escape from the sinus. (Ambroise Paré described this feature of " open " extradural abscess.)

He had no symptoms except a mild local headache each time the sinus was about to discharge.

In the period since the appearance of the cranial infection an ischiorectal abscess, and later a Brodie's abscess of the humerus, had been successfully treated.

On examination there were no neurological abnormalities. The patient was pale and rather "toxic" looking. A radiograph of the skull (Fig. 1) showed changes in

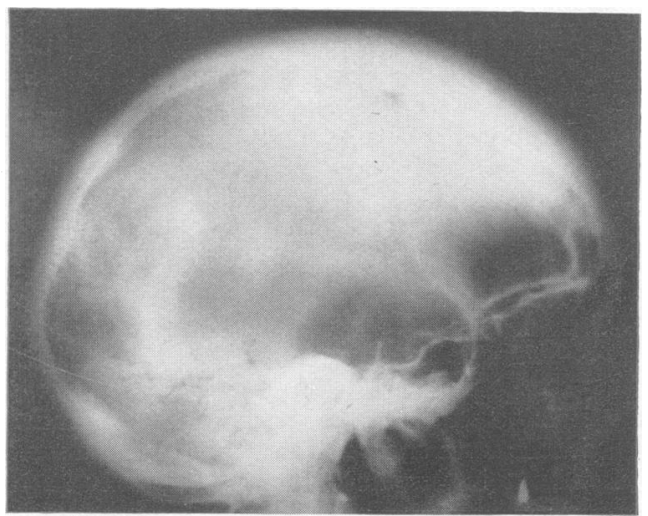

Fig. 1.-Appearance of the skull in Case 1. The thickening of calvarium extended only to the midline. the bone. The blood Wassermann and Kahn reactions were negative.

At operation in February, 1951, the sinus-track in the bone was found to be about $5 \mathrm{~mm}$. in diameter, with rounded margins. A rectangular block of calvarium about $25 \mathrm{~mm}$. thick and $9 \mathrm{~cm}$. square was removed, exposing almost the whole of a chronic extradural abscess. In making the saw-cuts a bevelling of about $45^{\circ}$ was arranged so as to minimize "dead-space " on closure. The bone was dense, fairly hard and there was no indication of diploe.

The extradural abscess extended a short distance inferiorly towards the supraorbital ridge, and bone was nibbled away to expose fully the abscess cavity. The wound was closed in two layers, with three gutta-percha tissue drains which were later shortened, and removed on the eighth day. Systemic penicillin and sulphamezathine were given and healing was satisfactory.

Two organisms were recovered on culture: Staph. albus and $\beta$-haemolytic streptococcus, both sensitive to penicillin and streptomycin, but resistant to sulphonamides. It may be significant that sulphonamides had been used in this man's infection of the humerus and peri-rectal tissues.

Eight months later the bone flap, which had been stored sterile, was re-inserted, provision being made for the instillation of penicillin. Two months later clear, yellow, sterile fluid was aspirated from under the scalp flap. There was no pyrexia.

There has been no sign of recurrence of infection anywhere in the three years to date, although we had initially some anxiety as to how the tissues would receive this substantial block of dead bone.

Case 2.-This was a woman aged 33 years. She had a 31 years' history of recurrent cranial infection, beginning with a scalp abscess near the vertex, following pneumonia at the age of 2 years. The abscess was incised and later healed. The family then moved overseas. At the age of 10 years this patient was assailed by a serious malady with marked pyrexia, rigidity of the limbs, and unconsciousness; it was not thought that she would recover. Lumbar puncture did not confirm the diagnosis of "meningitis". (Details of this illness are not procurable.) She recovered, contrary to all expectations.

Some months later an abscess of the scalp began to discharge, and continued to do so intermittently over the years, each episode of discharge being preceded by headache at the vertex. Horizontal diplopia was noted on one such occasion. 


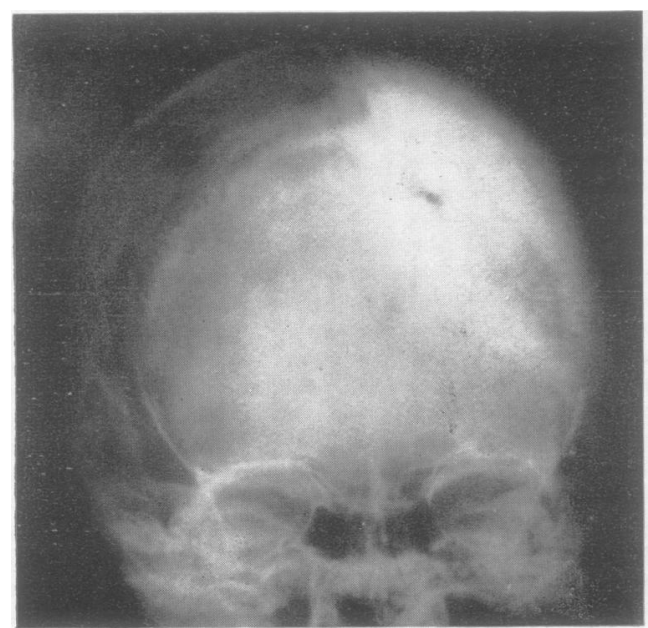

Fig. 2.-Appearance of the skull in Case 2.

The sinus was opened and curetted when the patient was 18 years old.

Twelve years ago she came under neurosurgical care in this country, with a discharging sinus above the left ear. Papilloedema was present. A burr-hole intended for ventriculography released $55 \mathrm{ml}$. of extradural pus. The cavity was drained for six weeks, and all three foci of drainage soon closed.

All was well for five years, then the parieto-occipital sinus again opened and discharged for months at a time, at long intervals. Local throbbing headache preceded each breakdown.

On examination she looked well. There was a small sinus near the midline and behind the vertex which had been discharging green pus continuously for seven months (dressed thrice daily). The scalp was reddened and oedematous, but not tender, for some $4 \mathrm{~cm}$. wide of the sinus all round. Nearby posteriorly were some operation scars where the scalp was thin and fixed. The regional lymph glands were not palpable. There was no pyrexia nor any abnormal neurological signs. The blood Wassermann and Kahn reactions were negative. The $x$-ray appearance is shown in Fig. 2.

At operation in October, 1953, block resection of diseased calvarium over an area of 15 by $18 \mathrm{~cm}$. revealed an extensive indolent extradural abscess, with shaggy granulations but little pus. The margins of the craniectomy were cut with a pronounced bevel, as in Case 1 . The endocranial aspect of the bone-flap was extremely irregular but not eroded. The inner table bore incrustations and irregularities, but its surface was intact (Fig. 3). The dura mater was extremely thickened and semi-rigid, except peripherally beyond the sharply delimited abscess.

The craniectomy extended to the midline superiorly, and bleeding from the sagittal sinus was controlled with gelatine foam later removed lest it form a nidus for infection. Sulphanilamide powder was insufflated and the scalp closed with one layer of sutures. Two guttapercha tissue drains and a tube for instillation of peni-

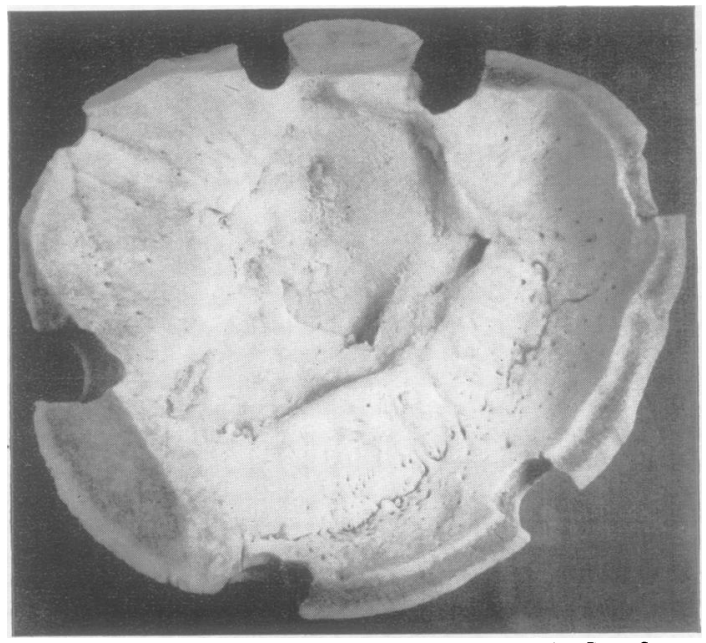

FIG. 3.-Endocranial aspect of the bone flap in Case 2.

cillin were provided ; these were removed after two days $\overrightarrow{.}$ Penicillin was instilled daily, 50,000 to 100,000 units at a time, for five days. Aureomycin was given thereafterfor several days. Healing was rapid, but a small amount of serous discharge persisted at the old unhealthy sinuso area. At operation the scalp here was found to be thin? and devitalized over an area too extensive to permitos excision and suture.

The cultures from a deep field at operation we sterile, and no organisms were seen in films. Lafief cultures from the persisting localized sinus grew Staph pyogenes, universally sensitive.

We had contemplated re-insertion of the sterile stores bone flap, which weighed $376 \mathrm{~g}$., but that was impracs ticable because of the risk presented by the thin arep of scalp. Moreover, as pointed out by King (1937), ir the more acute osteitis regeneration of bone afte 8 craniectomy is to be anticipated. Incidentally, experience has shown that the misfortune of operative infection around bone grafts may ultimately promote abundant union.

Six months after craniectomy the thin area of scalp. was widely resected and a large sliding graft was swung over from the right side.* We saw no evidence of recurrent suppuration wide of the small sinus at this second operation. We believe it would be bad surgers. to insert the bone flap now in this wound, even werg it desirable.

Recent $x$-ray films, taken a year after the craniectomy show several foci of re-ossification at dural level. The scalp is in excellent condition.

Case 3.-The patient, a man aged 32 years, sustained a non-concussional blow with a cricket ball on the right temple when he was 9 years old. He suffered from headache for a short time afterwards, but attended school without interruption. Two weeks later he hag

* We wish to acknowledge gratefully the assistance of Mr. G. Mo FitzGibbon and Mr. Routledge of this hospital in planning the incisions for this plastic repair. 


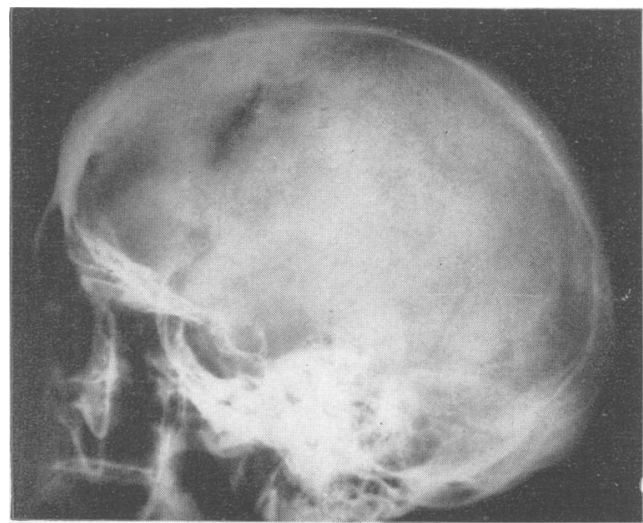

FIG. 4.-Appearance of the skull in Case 3. Hyperostosis is external; note the even contour of the inner table. $B y$ courtesy of Dr. A. R. Thomas.

a sudden "black-out", but no convulsion and no incontinence. A similar brief attack followed three days later. The patient was admitted to the local hospital where he had an operation on the following day. (No details now procurable.) He remained in hospital for nine months, and at intervals eight operations were performed through short incisions. Small sequestra were extruded spontaneously from time to time. $\mathrm{He}$ then attended as an out-patient for four years on account of a discharging sinus at the right temple. During this time he had recurrent headaches but no febrile symptoms.

He remained well for 14 years, then had a sudden attack of unconsciousness without overt epileptic phenomena. Thereafter, he had recurrent right frontal headaches for a few days at a time. There was no vomiting or pyrexia, and no neurological symptoms.

Nine weeks before he came under our care he developed a red, tender, hot swelling near the midline of the forchead, which was incised after a short course of penicillin; no pus or organisms were found. He was febrile at the onset. The wound quickly healed and the headaches ceased.

Examination showed a fit-looking man. There were eight small scars in the temporal region and near the midline of the forehead; the skin was thin and adherent to the bone at some of the scars. The thickening of the frontal bone terminated rather abruptly at about the level of the coronal suture, where a "step " was palpable. This was in contrast to the foregoing cases, where no indication of the limits of hyperostosis was detectable by palpation. There were no abnormal neurological findings. The blood Wassermann reaction was negative, and the cerebrospinal fluid was normal at a pressure of $150 \mathrm{~mm}$. The $x$-ray appearances are shown in Fig. 4 ; the frontal air sinuses appeared healthy.

At operation in December, 1953, an intertemporal incision was chosen to provide a large frontal scalp flap. The skull presented a diffuse right frontal and median bossing, and the surface was extensively roughened and pitted, the fibrous tissue of the thick periosteum dipping down into these pits (Fig. 5). Five burr-holes

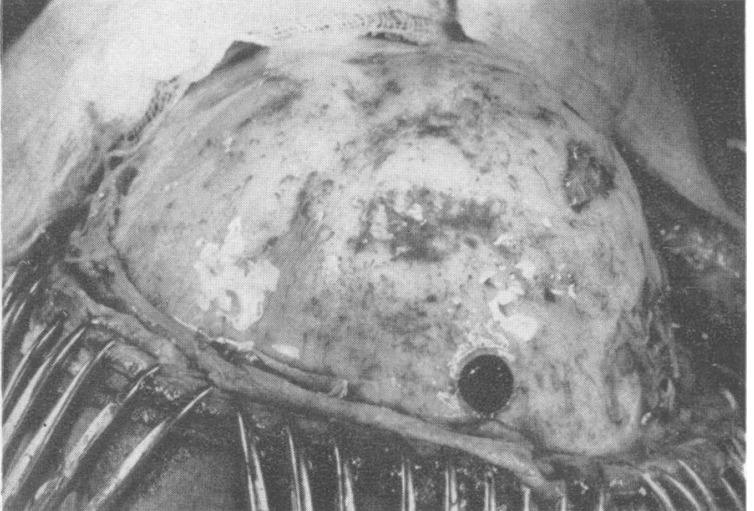

FIG. 5.-Appearance at operation in Case 3. The burr-hole lies posteriorly.

made in the right temporal region and three large drill holes near the frontal midline yielded no pus ; the visible dura was normal. The wound was closed without drainage. Mild oedema of the scalp persisted for some days, and sterile fluid accumulating under it was aspirated on two occasions. Penicillin was administered during operation and subsequently.

In the year since operation this patient has had two attacks of headache, each associated with coryza. These have cleared with symptoms suggesting the resolution of an obstruction in one frontal sinus.

\section{Discussion}

This hyperostotic reaction of the calvarium to chronic pyogenic infection must be somewhat rare. It is, however, possible that conditions of the skull akin to this may appear sporadically in the future where intensive systemic treatment of acute osteitis of the skull with penicillin has just failed to stamp out the infection completely. In this connexion the long clinical remissions in the cases described may be pertinent. And indeed time may perhaps show that we have been less successful with these cases than we believe.

The only relevant paper which we have found in the literature is one by Maxwell (1946) describing chronic proliferative osteitis affecting the base of the skull and adjacent calvarium following frontal sinusitis treated by local operations. Follow-up is not recorded.

It is to be noted that the hyperostosis extended well beyond the limits of the extradural abscesses and that the bone showed no gross evidence of infection. The extent of the hyperostosis in each case corresponded to a suture line at one or more of its boundaries. The term osteitis applied to this condition might therefore be questioned, but it seems to us convenient. The hyperostosis appeared to be 
a reaction of healthy bone to adjacent chronic infection. The surface of bone contiguous with the focus of suppuration in each case showed irregularities but no necrosis or hint of sequestration at an earlier stage. Sequestra were discharged in the initial subacute phase in Case 3 , and there was undoubtedly a grumbling osteitis of the outer table. In that particular case we have no accurate information as to the presence of a septic cut or abrasion after the injury, but the infection probably came from without.

In two patients the cranial infection appeared in childhood, but the similar findings in Case 1 preclude a deduction that infection arising during the growth period is a factor in the pathology.

Acute infection in bone is associated with hyperaemia and leads to osteolysis. Chronic infection causes osteosclerosis, and probably surrounding scar formation induces a relatively anoxaemic state locally in the affected bone tissue. Even so, the extent of the hyperostosis beyond the obvious focus in the cases described is a feature of interest. Incidentally, a good case has been made out for chronic local hypoxia as the basis for deposition of calcium in the cerebral cortex by Alexander and Woodhall (1943) and the same circumstance probably accounts for the cortical calcifications in the Sturge-Weber anomaly "(personal observations in progress).
The headache in the first two cases was probably of dural origin. In Case 3 it was described by the patient in terms which suggested the pain of periostitis ; it was relieved by local surgery in his earlier years, and our fruitless exploration involving. extensive stripping of periostium was promptly effective. The behaviour of this man's headache in years to come will be of interest.

Infection apparently did not spread transdurally if any case. Our interpretation of the scanty informa tion about the serious malady in childhood isō Case 2 is that the patient was in peril from the effects of brain-stem compression occasioned by a substantial extradural abscess.

Regarding treatment, it is obvious that th $\vec{\oplus}$ principles accepted in the surgery of acute osteitis of the skull must be applied to these chronif infections. Small local drainages cannot cure chronic extradural abscess. The ability of cocci te remain dormant for long periods in avascular tissue is notorious. We found no pus in Case 3 and therefore did not perform craniectomy.

We wish to express our indebtedness to the laboratory staff, and to Mr. Banham, of the photographic departo ment of Frenchay Hospital, for their help with the investigations and records.

\section{REFERENCES}

Alexander, L., and Woodhall, B. (1943). J. Neuropath., 2, 1. King, J. E. J. (1937). Surgery, 1, 401. Maxwell, J. H. (1946). Ann. Otol., St. Louis, 55, 719. 